\title{
Freezing of hard spheres in confinement
}

\author{
Willem K. Kegel ${ }^{\text {a) }}$ \\ Van't Hoff Laboratory for Physical and Colloid Chemistry, Debye Institute, Utrecht University, \\ Padualaan 8, 3584 CH Utrecht, The Netherlands
}

(Received 4 June 2001; accepted 19 July 2001)

\begin{abstract}
The influence of confinement on the freezing transition of hard spheres is investigated. Two limiting cases are considered: (1) large systems, where walls weakly perturb the bulk system, and (2) small systems where the influence of geometry becomes important. In the first situation, the shift in coexisting densities is a linear function of the area to volume ratio in the system. This is a manifestation of the Kelvin equation, and the phenomenon is thermodynamically equivalent to capillary condensation. A claim (by others) of "prefreezing" of hard spheres at a smooth hard wall is quantitatively attributed to capillary crystallization. It is shown that the coexistence region narrows as a function of the area to volume ratio. In the second limit two different confined geometries are studied. In these limits, widening of the coexistence region is observed, pointing to an upper and lower critical point at intermediate values of the area to volume ratio, or no critical point at all. In a slit geometry buckling transitions interfere with the freezing transition. In a box geometry, at large values of the area to volume ratio, fluctuations become important. These fluctuations determine the fate of the freezing transition at intermediate values of the area to volume ratio. () 2001 American Institute of Physics. [DOI: 10.1063/1.1401825]
\end{abstract}

\section{INTRODUCTION}

The understanding of phase transitions and critical phenomena in some classes of homogeneous bulk systems were triumphs of nineteen and twentieth century physics. ${ }^{1}$ However, truly homogeneous bulk systems hardly exist in nature. Most systems are either confined, like porous stone, catalysts, and biological cells, or are otherwise in contact with surfaces. These surfaces may either be structured or smooth, and will interact with the molecules in the system. The presence of surfaces has been known for a long time to significantly alter the thermodynamics and dynamics of phase transitions, a dramatic example being the role of dust in the formation of liquid in supersaturated vapor. Dust particles lower the free energy barrier of a liquid water nucleus in its supersaturated vapor, leading to nucleation rates that are many orders of magnitude larger than those in a homogeneous system, see, for example, Ref. 2.

Much work has been done on layering transitions of molecular systems at walls, theoretically ${ }^{3}$ as well as experimentally. ${ }^{4}$ Condensation in pores (cylinders, slits), see for example Refs. 5, 6, and freezing and melting in pores, ${ }^{7,8}$ have been the subject of intense study, see also Refs. 9, 10 for reviews. In these systems, molecules and walls interact by both attractive and excluded volume interactions. In the experimental systems it is usually not clear how (i.e., by what potential) molecules interact with the walls. Moreover, popular experimental model systems such as Vycor and silica xerogel do not have a simple pore geometry. This makes it difficult to compare experiments to theory, as was indeed concluded in the recent review paper by Gelb and co-workers. ${ }^{9}$

${ }^{a)}$ Electronic mail: W.K.Kegel@chem.uu.nl
In this work the question is addressed as to what confinement does to the freezing transition of hard spheres, where the confining surfaces and the spheres interact by excluded volume interactions. Hard spheres under thermal agitation have been (and still are) vigorously studied. In bulk they undergo a first-order phase transition from a fluid to a crystal (referred to as the freezing transition) at number densities significantly below the density where the system is close-packed, as was found by computer simulation ${ }^{11-13}$ as well as by experiments on colloids. ${ }^{14}$ Indeed colloidal systems can be tailored so as to behave as hard spheres. ${ }^{15}$ In principle it is possible to design well-defined pore geometries with (super) colloidal size, say on the order of micrometers and larger, by using lithographic techniques. Therefore, predictions made in this work can experimentally be verified by using colloids in between slits, or in other geometries. The surfaces of these geometries should be treated in such a way that only excluded volume interactions operate between the spheres and the confining walls.

Two limiting cases are considered in this work. In the first case (Sec. II) walls are treated as small perturbations of a bulk system, i.e., the total free energy of the system is the bulk free energy plus a correction that is linear in $A / V$, with $A$ the area of the wall(s) and $V$ the system volume. This approach leads to a general expression for the shift in coexisting densities of first-order phase transitions of systems in contact with walls, at constant volume. This expression essentially resembles the Kelvin equation, but here emphasis is on density shifts with respect to the bulk, and not on chemical potential shifts. It will be shown that this result is generally valid for (effectively) single component systems where the range of interparticle interactions is much smaller than the linear system size. Subsequently, attention is turned to hard spheres in contact with smooth hard walls (Sec. II B). 
Depending upon the wall-crystal face $(111,110$ or 100) contact, the coexistence region shifts to lower densities or higher densities as a function of the area to volume ratio in the system. Independent of which crystal face contacts the wall, the melting and freezing lines converge. Extrapolation, beyond the limit where the walls act as a weak perturbation of the bulk system, indicates a critical point. In Sec. II C, "prefreezing" is discussed, i.e., formation of one or more crystal layers at a smooth hard wall at small undersaturations, in the light of the results presented in Sec. II B.

In the other limiting case, treated in Sec. III, the area to volume ratio is large. Two different geometries are considered. The first is a slit (between plates) geometry where the area to volume ratio may become large, but the number of particles still goes to infinity, and the system always is in the thermodynamic limit. Extrapolation of the coexistence region in the capillary approximation indicates a critical point, but computer simulations by others reveal that before this point is reached, buckling transitions come into play. The second geometry is a box. The analysis is performed in the grand ensemble, concentrating on the properties of the peaks of the grand distribution function. It turns out that in this situation the melting and freezing lines diverge with increasing $A / V$. In this limit, at large $A / V$, fluctuations play an important role. In fact, depending upon how the coexistence lines cross over from their behavior at small $A / V$, to their behavior at large $A / V$, fluctuations may kill the freezing transition in some interval of $A / V$.

\section{SMALL AREA TO VOLUME RATIOS: CAPILLARY LIMIT}

The scheme here is to find a relevant reference free energy that only depends on bulk properties, subsequently add a surface contribution to it, and then solve for the coexisting densities. This leads to a general relation between the shift of coexisting densities and properties of the system defined at bulk-coexistence conditions (Sec. II A). In Sec. II B, the results are applied to hard spheres in contact with hard walls, the relevant parameters being extracted from equations of state, theories and (simulation) data from the literature.

\section{A. Shift of coexisting densities as a function of area to volume ratio}

A first-order phase transition in single (effective) component systems can be seen as two branches of the free energy as a function of density having a common tangent at the coexisting densities. In this situation it is always possible to add a linear contribution (in density) to the free energies such that the coexisting densities become minima of the free energies. This property will be pursued. First of all we concentrate on the free energy branches close to coexistence. Let $\phi_{1}$ and $\phi_{2}$ be the volume fractions of single (effective) components in phase 1 and 2 (the subscript indicates the identity of the phase, i.e., solid, liquid, or gas). Close to the coexisting (bulk) volume fractions, further denoted as $\phi_{1}^{*}$ and $\phi_{2}^{*}$ (the asterisk denotes properties of the bulk system), the reduced free energies of the two branches are written as

$$
\begin{aligned}
f_{1}=\frac{\beta F_{1} v}{V}= & f_{1}\left(\phi=\phi_{1}^{*}\right)+\left(\frac{\partial f_{1}}{\partial \phi}\right)_{\phi=\phi_{1}^{*}} \Delta \phi_{1} \\
& +\frac{1}{2}\left(\frac{\partial^{2} f_{1}}{\partial \phi^{2}}\right)_{\phi=\phi_{1}^{*}} \Delta \phi_{1}^{2}+O\left(\Delta \phi_{1}\right)^{3}, \\
f_{2}=\frac{\beta F_{2} v}{V}= & f_{2}\left(\phi=\phi_{2}^{*}\right)+\left(\frac{\partial f_{2}}{\partial \phi}\right)_{\phi=\phi_{2}^{*}} \Delta \phi_{2} \\
& +\frac{1}{2}\left(\frac{\partial^{2} f_{2}}{\partial \phi^{2}}\right)_{\phi=\phi_{2}^{*}} \Delta \phi_{2}^{2}+O\left(\Delta \phi_{2}\right)^{3} .
\end{aligned}
$$

In these equations $F_{\alpha}$ is the Helmholtz free energy of the branch $\alpha, \Delta \phi_{\alpha}=\phi_{\alpha}-\phi_{\alpha}^{*}, \beta=1 / k T$ with $k$ Boltzmann's constant, and $T$ absolute temperature, $v=(\pi / 6) \sigma^{3}$, with $\sigma$ defined as the range of intermolecular interactions. In many cases this is not a well-defined quantity, but for hard spheres $\sigma$ clearly is the sphere diameter and $v$ the volume of a single sphere (not the molecular volume). $V$ is the system volume. The reason for writing the free energy in this (reduced) form has advantages that will become clear later-it has to do with a proper scaling of the interfacial area-volume ratio. Now a linear contribution to Eqs. (2.1a) and (2.1b) can be added such that the coexisting densities in the bulk, $\phi_{1}^{*}$ and $\phi_{2}^{*}$ become minima of the free energy. Therefore the relevant reference free energies in the bulk are (for not-too-great $\left.\Delta \phi_{\alpha}\right)$,

$$
\begin{gathered}
f_{1 b}=\frac{1}{2}\left(\frac{\partial^{2} f_{1}}{\partial \phi^{2}}\right)_{\phi=\phi_{2}^{*}} \Delta \phi_{1}^{2}=\frac{1}{2} u_{1 b} \Delta \phi_{2}^{2}, \\
f_{2 b}=\frac{1}{2}\left(\frac{\partial^{2} f_{2}}{\partial \phi^{2}}\right)_{\phi=\phi_{2}^{*}} \Delta \phi_{2}^{2}=\frac{1}{2} u_{2 b} \Delta \phi_{2}^{2} .
\end{gathered}
$$

In these equations the first subscript again assigns the identity of the phase ( 1 or 2$)$, and the second one (" $b$ ") refers to properties of the bulk system (i.e., without influence of walls). The reference free energies Eqs. (2.2) are shown schematically in Fig. 1 (curves 1 and 2). Equations (2.2) imply that the reference bulk system is approximated by a harmonic potential well with a strength given by the reduced bulk moduli,

$$
u_{\alpha b}=\left(\frac{\partial^{2} f_{\alpha}}{\partial \phi^{2}}\right)_{\phi=\phi_{\alpha}^{*}}=\left(\frac{\partial \widetilde{\mu}_{\alpha}}{\partial \phi}\right)_{\phi=\phi_{\alpha}^{*}}=\left(\frac{1}{\phi} \frac{\partial \widetilde{p}_{\alpha}}{\partial \phi}\right)_{\phi=\phi_{\alpha}^{*}},
$$

with the reduced chemical potential in phase $\alpha(\alpha$ $\in\{1,2\}): \widetilde{\mu}_{\alpha}=\beta \mu_{\alpha}$ and the reduced pressure of phase $\alpha$ : $\widetilde{p}_{\alpha}=\beta p_{\alpha} v$. The corresponding symbols without tilde refer to the unreduced quantities.

The next step is to add surface contributions to Eqs. (2.2). The reduced surface free energy is written as

$$
f_{\alpha s}=\tilde{\gamma}_{\alpha} * a_{v},
$$

where $\tilde{\gamma}_{\alpha}=\beta \sigma^{2} \gamma_{\alpha}, \gamma_{\alpha}$ denoting the interfacial tension between phase $\alpha$ and the wall. The subscript "s" refers to "surface," while $\alpha$ again refers to one of the coexisting 


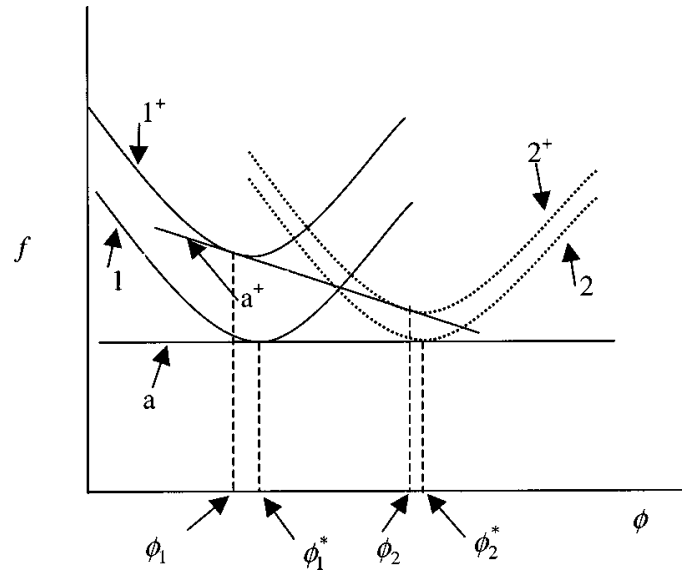

FIG. 1. Schematic view of the two branches of the (reduced) free energy, and their common tangents. The branches 1,2 are the bulk reference free energies, Eqs. (2.2). The line $a$ indicates the common tangent of the reference branches at the coexisting bulk volume fractions $\phi_{1}^{*}$ and $\phi_{2}^{*}$. The branches $1^{+}, 2^{+}$are equal to the branches 1,2 , but with a surface contributions added. This situation resembles Eqs. (2.6), with the special situation that here, $u_{1 s}=u_{2 s}=0$. The line designated as $a^{+}$is the new common tangent at the points $\phi_{1}=\phi_{1}^{*}+\Delta \phi_{1}$, and $\phi_{2}=\phi_{2}^{*}+\Delta \phi_{2}$, where the $\Delta \phi$ 's are given by Eqs. (2.10), being the solutions of Eqs. (2.7)-(2.9).

phase. The quantity $a_{v}$ denotes the reduced area to volume ratio. It relates to the total area, $A$, and volume, $V$, of the system by

$$
a_{v}=\frac{A / \sigma^{2}}{V / v} .
$$

This implies that $a_{v} \propto \sigma / L$, with $L$ the linear size of the system. Now $a_{v}$ will later be treated as the small parameter, implying that the range of interactions be small compared to the (linear) system size. The interfacial tension will in general depend upon density. This dependence is again written as a power series in $\Delta \phi_{\alpha}$ around $\phi_{\alpha}^{*}$. It turns out that higher than first order contributions in $\Delta \phi_{\alpha}$ only show up in the final result as terms that are quadratic in $a_{v}$ and these terms are neglected as, for example, line and curvature contributions to the surface free energy will also lead to order $a_{v}^{2}$ terms. Therefore, denoting $\widetilde{\gamma}_{\alpha}^{*}$ as the interfacial tension of phase $\alpha$ when its density equals $\phi_{\alpha}^{*}$, we may write

$$
\widetilde{\gamma}_{\alpha}=\widetilde{\gamma}_{\alpha}^{*}+\left(\frac{\partial \widetilde{\gamma}_{\alpha}}{\partial \phi}\right)_{\phi=\phi_{\alpha}^{*}} \Delta \phi_{\alpha}=\widetilde{\gamma}_{\alpha}^{*}+u_{\alpha s} \Delta \phi_{\alpha},
$$

with $u_{\alpha s}=\left(\partial \gamma_{\alpha} / \partial \phi\right)_{\phi=\phi_{\alpha}^{*}}$. Adding this contribution to the bulk free energy, Eq. (2.2), leads to the free energy branches of a system in contact with walls,

$$
\begin{aligned}
& f_{1}=\frac{1}{2} u_{1 b} \Delta \phi_{1}^{2}+\left(\widetilde{\gamma}_{1}^{*}+u_{1 s} \Delta \phi_{1}\right) a_{v}, \\
& f_{2}=\frac{1}{2} u_{2 b} \Delta \phi_{2}^{2}+\left(\widetilde{\gamma}_{2}^{*}+u_{2 s} \Delta \phi_{2}\right) a_{v} .
\end{aligned}
$$

These branches are schematically indicated in Fig. 1 as $1^{+}$ and $2^{+}$. In order to find the shifts in coexisting densities, $\Delta \phi_{1}$ and $\Delta \phi_{2}$ we need to find the common tangent of Eqs. (2.6a) and (2.6b) (also indicated in Fig. 1) which is analogous to solving

$$
\widetilde{\mu}_{1}=\widetilde{\mu}_{2} \text { and } \widetilde{p}_{1}=\widetilde{p}_{2},
$$

with

$$
\begin{aligned}
\tilde{\mu}_{1} & =\left(\frac{\partial f_{1}}{\partial \phi}\right)_{T, \phi=\phi_{1}}=u_{1 b} \Delta \phi_{1}+u_{1 s} a_{v}, \\
\tilde{\mu}_{2} & =\left(\frac{\partial f_{2}}{\partial \phi}\right)_{T, \phi=\phi_{2}}=u_{2 b} \Delta \phi_{2}+u_{2 s} a_{v}, \\
\widetilde{p}_{1} & =-f_{1}+\phi_{1}\left(\frac{\partial f_{1}}{\partial \phi}\right)_{T, \phi=\phi_{1}} \\
& =\frac{1}{2} u_{1 b} \Delta \phi_{1}^{2}+u_{1 b} \phi_{1}^{*} \Delta \phi_{1}+\left(u_{1 s} \phi_{1}^{*}-\widetilde{\gamma}_{1}^{*}\right) a_{v}, \\
\widetilde{p}_{2} & =-f_{2}+\phi_{2}\left(\frac{\partial f_{2}}{\partial \phi}\right)_{T, \phi=\phi_{2}} \\
& =\frac{1}{2} u_{2 b} \Delta \phi_{2}^{2}+u_{2 b} \phi_{2}^{*} \Delta \phi_{2}+\left(u_{2 s} \phi_{2}^{*}-\widetilde{\gamma}_{2}^{*}\right) a_{v},
\end{aligned}
$$

for $\Delta \phi_{1}$ and $\Delta \phi_{2}$. Equations (2.8) follow from

$$
\frac{v}{V} \tilde{\mu}=\left(\frac{\partial f}{\partial N}\right)_{T, V}=\left(\frac{\partial f}{\partial \phi}\right)_{T}\left(\frac{\partial \phi}{\partial N}\right)_{V}=\frac{v}{V}\left(\frac{\partial f}{\partial \phi}\right)_{T},
$$

and Eqs. (2.9) from

$$
\begin{aligned}
\tilde{p} & =-\left(\frac{\partial(\beta F v)}{\partial V}\right)_{T, N}=-\left(\frac{\partial(V f)}{\partial V}\right)_{T, N} \\
& =-f-V\left(\frac{\partial f}{\partial \phi}\right)_{T}\left(\frac{\partial \phi}{\partial V}\right)_{N}=-f+\phi\left(\frac{\partial f}{\partial \phi}\right)_{T} .
\end{aligned}
$$

For $a_{v} \ll 1$ (implying $\sigma / L \ll 1$ ), and again neglecting terms of order $a_{v}^{2}$ we get from Eqs. (2.7) to (2.9),

$$
\begin{aligned}
& \Delta \phi_{1}=\left(\frac{\left(\tilde{\gamma}_{2}^{*}-\widetilde{\gamma}_{1}^{*}\right)}{u_{1 b}\left(\phi_{2}^{*}-\phi_{1}^{*}\right)}-\frac{u_{1 s}}{u_{1 b}}\right) a_{v}, \\
& \Delta \phi_{2}=\left(\frac{\left(\tilde{\gamma}_{2}^{*}-\widetilde{\gamma}_{1}^{*}\right)}{u_{2 b}\left(\phi_{2}^{*}-\phi_{1}^{*}\right)}-\frac{u_{2 s}}{u_{2 b}}\right) a_{v} .
\end{aligned}
$$

This result has clear geometrical significance, see again Fig. 1. As for the first terms in Eqs. (2.10), $\left(\widetilde{\gamma}_{2}^{*}-\widetilde{\gamma}_{1}^{*}\right)$ quantifies the shift of the reference free energy parabola relative to each other in the vertical direction in the free energy-density plane, i.e., the shift of the curves 1 and 2 to $1^{+}, 2^{+}$in Fig. 1. The larger its magnitude (positive or negative), the greater the shift in coexisting densities. The shift becomes larger the smaller the curvature of the parabola and/or the smaller the width of the coexistence region. The second terms take into account the distortion of the parabola by the density dependence of the interfacial tension between the phases 1 or 2 and the wall. If a positive linear contribution is added to one of the parabola (or different linear contributions to both), the common tangent points shift to lower densities, the magnitude of the shift being determined by the ratio of the linear contribution and the curvature of the parabola. The second terms resemble the adsorption densities at the wall, $\Gamma_{1}$ and $\Gamma_{2}$, since 


$$
\Gamma_{\alpha}=-\left(\frac{\partial \gamma_{\alpha}}{\partial \mu_{\alpha}}\right)_{T}=-\left(\frac{\partial \gamma_{\alpha}}{\partial \phi}\right)_{T}\left(\frac{\partial \phi}{\partial \mu_{\alpha}}\right)_{T}=-\frac{u_{\alpha s}}{u_{\alpha b}}
$$

so that Eqs. (2.10) may also be written as

$$
\begin{aligned}
& \Delta \phi_{1}=\left(\frac{\left(\tilde{\gamma}_{2}^{*}-\tilde{\gamma}_{1}^{*}\right)}{u_{1 b}\left(\phi_{2}^{*}-\phi_{1}^{*}\right)}+\Gamma_{1}\right) a_{v}, \\
& \Delta \phi_{2}=\left(\frac{\left(\tilde{\gamma}_{2}^{*}-\tilde{\gamma}_{1}^{*}\right)}{u_{2 b}\left(\phi_{2}^{*}-\phi_{1}^{*}\right)}+\Gamma_{2}\right) a_{v} .
\end{aligned}
$$

It should be noted that the location of the dividing surface may always be chosen in such a way as to make (one of the) adsorption densities equal to zero (the Gibbs dividing surface). However, in this case such a choice would be awkward and unphysical, leading to dividing surfaces that may be far away from the walls.

Equations (2.10)-(2.11) apply to atomic and colloidal systems, and also to metastable equilibria, in the limit that the range of interactions be small compared to the system size, or $a_{v}$ goes to zero. This limit will further be referred to as the capillary limit. Only systems with pathologically longranged interactions will never reach this limit.

In ending this section we note that Eqs. (2.11) are analogous to the Kelvin equation. This equation shows up in the literature in various disguises, see for example, Ref. 16 for a nice discussion. In its most general form it relates the shift of the chemical potential at coexistence to the mean radius of curvature of the interface between the coexisting phases. Inserting Eqs. (2.10) into Eqs. (2.8) for a system in a slit between two plates separated by a distance $L$, and realizing that the reduced chemical potential Eqs. (2.8) is in fact the negative of the undersaturation, i.e., $\tilde{\mu}=\left(\mu-\mu^{*}\right) / k T$ $=-\Delta \mu / k T$ (the asterisk again refers to the bulk system) leads to the well-known Kelvin equation, ${ }^{17}$

$$
\Delta \mu=\left(\frac{-2\left(\gamma_{2}^{*}-\gamma_{1}^{*}\right)}{\left(\rho_{2}^{*}-\rho_{1}^{*}\right)}\right) \frac{1}{L}=\left(\frac{2 \gamma_{12}^{*} \cos \theta}{\left(\rho_{2}^{*}-\rho_{1}^{*}\right)}\right) \frac{1}{L} .
$$

In this equation the $\rho$ 's are the number densities in the coexisting phases, and in writing the last equality use has been made of Young's equation for the contact angle, $\theta$, at a single

wall $\gamma_{1}=\gamma_{2}+\gamma_{12} \cos \theta$. Note that $\gamma_{1}$ usually is associated with the tension of the solid-gas interface, $\gamma_{2}$ with the solidliquid interface, and $\gamma_{12}$ with the liquid-gas interface. For hard spheres in contact with a wall, the last mentioned tension is between the fluid and the crystal phase, and in principle is different for different crystal planes. There is a much easier way of obtaining Eq. (2.12) compared to the derivation presented here, see again Ref. 16, where it was derived in just a few steps starting from the grand potential. Can our main result, Eqs. (2.11), be derived starting from the more easily obtainable Eq. (2.12)? The shift in coexisting densities immediately follows from Eq. (2.12), via

$$
\Delta \phi_{\alpha}=-\left(\frac{\partial \phi_{\alpha}}{\partial \mu}\right) \Delta \mu=-\Delta \mu / u_{a b},
$$

but this leads to the shift in the bulk reference system [cf. Eq. (2.17), the density in the bulk where the confined system freezes] and not to the density within the confined (sub-) system itself. Indeed, this procedure does not naturally (i.e., as a consequence of collecting the linear contributions in the area to volume ratio) give rise to the adsorption terms in Eqs. (2.11).

\section{B. Shift of coexisting densities of hard spheres in contact with hard walls in the capillary limit}

In order to predict how the coexisting densities of hard spheres change with the surface-volume ratio in porous systems, we need Eqs. (2.10)-(2.11) together with values for the interfacial tensions of the fluid and the solid with the wall, the bulk moduli $u_{\alpha b}$, and the linear coefficients that take into account how the interfacial tensions vary with volume fraction, $u_{\alpha s}$. For the bulk part we take the equations of state by Hall. ${ }^{18}$ These pressures are in very good agreement with computer simulations, also at high densities, and the coexisting densities are accurate within the uncertainty range of computer simulations, see Ref. 19. These pressures read

$\widetilde{p}_{f b}=\frac{\phi_{f}\left(1+\phi_{f}+\phi_{f}^{2}-0.67825 \phi_{f}^{3}-\phi_{f}^{4}-0.5 \phi_{f}^{5}-1.7 \phi_{f}^{6}\right)}{1-3 \phi_{f}+3 \phi_{f}^{2}-1.04305 \phi_{f}^{3}}$,

$$
\widetilde{p}_{s b}=\frac{\phi_{s}\left(1+\phi_{s}+\phi_{s}^{2}-0.67825 \phi_{s}^{3}-\phi_{s}^{4}-0.5 \phi_{s}^{5}-6.028 \phi_{s}^{6} \exp \left[\left(y-\phi_{s}\right)\left(7.9-3.9\left(y-\phi_{s}\right)\right)\right]\right)}{1-3 \phi_{s}+3 \phi_{s}^{2}-1.04305 \phi_{s}^{3}},
$$

with $y=\left(\pi / 3 \sqrt{2} \phi_{s}\right)^{1 / 3}$. From Eqs. (2.13) and (2.14) we obtain the bulk quantities

$$
u_{\alpha b}=\left(\frac{\partial^{2} f_{\alpha}}{\partial \phi^{2}}\right)_{\phi=\phi_{\alpha}^{*}}=\left(\frac{\partial \widetilde{\mu}_{\alpha}}{\partial \phi}\right)_{\phi=\phi_{\alpha}^{*}}=\left(\frac{1}{\phi} \frac{\partial \widetilde{p}_{\alpha}}{\partial \phi}\right)_{\phi=\phi_{\alpha}^{*}}
$$

with $\alpha=f$ (fluid) or $\alpha=s$ (solid) listed in Table I.

Surface tensions between the fluid and the wall were calculated from scaled particle theory (SPT) using the general expression, ${ }^{20}$

$$
\widetilde{\gamma}_{f}=\frac{-9 \phi_{f}^{2}\left(1+\phi_{f}\right)}{2 \pi\left(1-\phi_{f}\right)^{3}}+\frac{\sigma^{3}}{2} \beta p=\frac{-9 \phi_{f}^{2}\left(1+\phi_{f}\right)}{2 \pi\left(1-\phi_{p}\right)^{3}}+\frac{3}{\pi} \widetilde{p},
$$

where we insert the Hall equation of state $\widetilde{p}:=\widetilde{p}_{f b}$ given by Eq. (2.13). This expression yields $\tilde{\gamma}_{f}=1.88$ at $\phi_{f}=0.494$, being in fair agreement with the value of (1.99 \pm 0.18$)$ obtained by computer simulation by Heni and Löwen ${ }^{21}$ at the same volume fraction. Equation (2.15) was expanded up to linear order around the bulk freezing volume fraction of 
TABLE I. Relevant quantities that determine solid-fluid coexistence densities; Eqs. (2.11). The subscript "av" for the solid systems indicates quantities that are averaged over the three crystal orientations. In the lower part of the table the quantities $\Delta \phi_{f, k}$ stands for the shift in coexisting volume fractions between fluid and solid with crystal orientation $k=(111)$, (100), (110) or "average."

\begin{tabular}{lccc}
\hline \hline Quantity & Fluid & Solid & Combined \\
\hline$u_{\alpha b}$ & 108.836 & 78.3407 & \\
$\phi_{\alpha}^{*}$ & 0.49221 & 0.543859 & \\
$\tilde{\gamma}_{f}^{*}$ & 1.44489 & & -0.41489 \\
$\tilde{\gamma}_{s, 111}^{*}-\widetilde{\gamma}_{f}^{*}$ & & & +0.10330 \\
$\widetilde{\gamma}_{s, 100}^{*}-\widetilde{\gamma}_{f}^{*}$ & & & +1.08435 \\
$\widetilde{\gamma}_{s, 110}^{*}-\widetilde{\gamma}_{f}^{*}$ & & & \\
$\tilde{\gamma}_{s, \text { av }}^{*}-\widetilde{\gamma}_{f}^{*}$ & -0.08333 & & \\
$\Gamma_{f}$ & & -0.10063 & \\
$\Gamma_{111}$ & & -0.15525 & \\
$\Gamma_{100}$ & & -0.25866 & \\
$\Gamma_{110}$ & & -0.171515 & \\
$\Gamma_{\mathrm{av}}$ & & -0.2032 & \\
$\left(\Delta \phi_{f, 111}\right) / a_{v}$ & -0.1571 & -0.1297 & \\
$\left(\Delta \phi_{f, 100}\right) / a_{v}$ & -0.0650 & +0.0093 & \\
$\left(\Delta \phi_{f, 1 a 0}\right) / a_{v}$ & +0.1096 & -0.1079 & \\
$\left(\Delta \phi_{f, \text { av }}\right) / a_{v}$ & -0.0375 & & \\
\hline \hline
\end{tabular}

0.49221 . From this expansion the quantities $\tilde{\gamma}_{f}^{*}$ and $u_{f s}$ $=\left(\partial \widetilde{\gamma}_{f} / \partial \phi\right)_{\phi=\phi_{f}^{*}}$ are obtained. These quantities are (implicitly) listed in Table I.

For the interfacial tensions of the crystal faces with the hard wall, we used a cell theory put forward by Heni and Löwen. ${ }^{21}$ Their theory includes that neighboring particles undergo collective excursions from their lattice positions. Their theory, termed "cell theory with fixed neighbors (CTFN)" by the authors, is indeed in very good agreement with direct computer simulations by the same authors, in particular near the melting transition. The relevant expressions read

$$
\tilde{\gamma}_{s, k}=\frac{g_{k}-g(2-y)}{2 g_{k} g_{b} y^{2}(y-1)},
$$

where the subscript $s$ again refers to "solid," and $k$ stands for the orientation of the crystal to the wall; $k=(111),(100)$, or (110). The quantities in Eq. (2.16) are given by $g=1 / \sqrt{2}$; $g_{111}=\sqrt{3} / 2 ; g_{100}=1.0 ; g_{110}=\sqrt{2}$. Equation (2.16) was expanded up to linear order around the bulk melting volume fraction of 0.543859 . From this expansion the (three) quantities $\widetilde{\gamma}_{s, k}^{*}$ and $u_{s s, k}=\left(\partial \widetilde{\gamma}_{s, k} / \partial \phi\right)_{\phi=\phi_{s}^{*}}$ are obtained. These quantities are (implicitly, that is, they follow from $\Gamma$ and $u_{\alpha b}$ ) listed in Table I. The negative adsorption densities in the table are caused by a depletion layer at the surface with a thickness of $\sigma / 2$. In Fig. 2 the coexisting density shifts in the confined system are shown graphically.

In confined systems in contact with bulk (like two plates hanging in a bulk system, or porous material immersed in bulk fluid), freezing may occur while the bulk system still is in the fluid state. This situation is thermodynamically completely analogous to capillary condensation. ${ }^{17}$ In that case it follows from putting Eqs. (2.8) equal to zero, together with the definition of adsorption density above Eqs. (2.11), that the volume fraction in the bulk, $\phi_{\text {bulk }}$, is related to the one in the porous system, $\phi_{\text {pores }}$, by

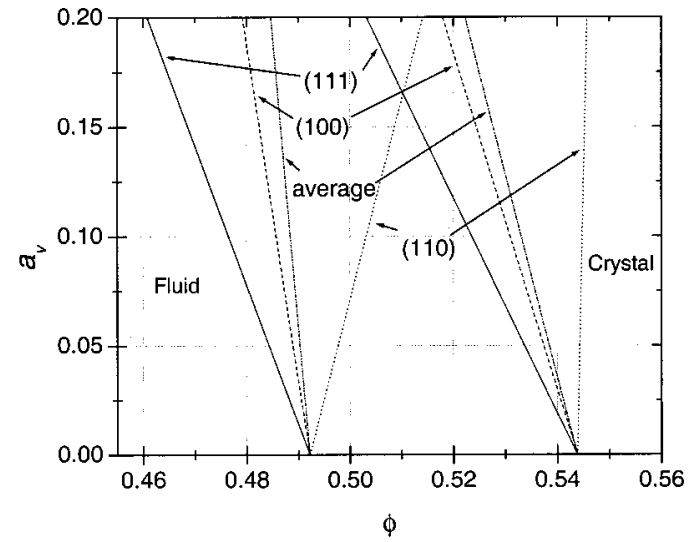

FIG. 2. Coexistence lines of hard spheres in pores in the volume fraction(reduced) area to volume ratio, $a_{v}$, plane. In each pair of lines, the ones on the left indicate freezing, and the ones on the right indicate melting. The situations for fluid-solid coexistence where the solid is oriented with its (111), (100), and (110) face along the walls are indicated in the figure. The lines indicated as "average" correspond to a situation where all three crystal orientations along the walls are equally distributed in the system.

$$
\phi_{\mathrm{bulk}}=\phi_{\mathrm{pores}}-\Gamma_{f} a_{v}=\phi_{f}^{*}+\left(\frac{\left(\widetilde{\gamma}_{s}^{*}-\widetilde{\gamma}_{f}^{*}\right)}{u_{f b}\left(\phi_{s}^{*}-\phi_{f}^{*}\right)}\right) a_{v} .
$$

The first equality of Eq. (2.17), valid for all fluid densities, states that the density in the pores being different from the density in the bulk is caused only by adsorbed material in the pores (being negative for hard spheres in contact with hard walls). From the second equality of Eq. (2.17), applicable at coexistence within the pores, it can be seen that if the interfacial tension between the solid and the wall is smaller than the one between the fluid and the wall, only then will material in the pores freeze while the bulk is still in a homogeneous fluid state, as is also obvious from Eq. (2.12). This is only the case for the (111) orientation of the crystal along the wall, see Table I. It is interesting to compare the behavior of confined molecular systems in this respect. Hydrogen, neon, oxygen, and argon confined in Vycor and silica xerogel, ${ }^{7}$ and alcohols in between mica surfaces ${ }^{8}$ and many other molecular systems in porous media ${ }^{9}$ freeze and melt at significantly lower temperatures than they do in the bulk. A lower freezing temperature at constant pressure, in most molecular systems, is analogous to a larger freezing density in systems at constant volume and temperature. Indeed, it follows from Eq. (2.12) that for a system confined in a slit of width $L$, the shift in freezing temperature relative to the one in the bulk, $\Delta T$, is given by (for not too great temperature shifts),

$$
\frac{\Delta T}{T}=\frac{-2\left(\gamma_{s}^{*}-\gamma_{f}^{*}\right)}{\lambda\left(\rho_{s}^{*}-\rho_{f}^{*}\right) L},
$$

with $\lambda$ the melting enthalpy in the bulk. This implies that in these molecular systems, the interfacial tension of the fluid with the walls is smaller than the one of all possible crystal faces with the walls. However, in a few cases increased freezing temperatures in confined systems have been observed, for the first time by Klein and Kumacheva, ${ }^{22}$ and 
later also by others, see Ref. 9, and references therein. This only seems to happen if (components in the) walls strongly attract the confined fluid molecules.

Now let us get back to our hard spheres. Looking at the densities within the confined system, it follows from the values listed in Table I and from Fig. 2 that depending upon the orientation of the crystal with the walls, the coexisting densities may either decrease (111 and 100 orientation) or increase (110 orientation) with the area to volume ratio. From the three possible orientations, the (111) orientation leads to the smallest increase in free energy compared to the bulk free energy. Therefore the thermodynamically stable crystal orientation along the walls is along the (111) direction. The coexisting densities in that case significantly decrease with the area to volume ratio: down to at least half the width of the bulk coexistence region. When the systems become small, then the gain in free energy by the fortunate (111) orientation will compete with the free energy cost of defects in the crystal-in most geometries, single crystals with the same orientation along all walls are impossible unless there are defects in the crystal. Only in some special (although extensively studied) cases, like a slit geometry, the crystalwall contact will be along the (111) crystal plane.

\section{Capillary crystallization and "prefreezing"}

It is quite interesting in the light of the previous section to pay a little attention to the phenomenon that was termed "prefreezing," as observed using molecular dynamics simulation by Courtemanche and co-workers, see Refs. 23, 24. Prefreezing is defined by these authors as the formation of one or more crystal layers of hard spheres at smooth hard walls at pressures below saturation pressure. We claim that this "prefreezing" is a manifestation of capillary crystallization. Indeed the lowest density where "prefreezing" was observed in Ref. 24 was at $98.6 \%$ below the saturation density, corresponding to a shift of the freezing volume fraction $\Delta \phi_{f}=-0.0069$. The authors employed a slit geometry with plate distance $L / \sigma=41.51$, corresponding to $a_{v} \approx 0.0482$. Now taking the value in Table I for the fluid branch, we predict at which volume fraction capillary crystallization occurs under these circumstances, i.e., at $\Delta \phi_{f, 111}$ $=-0.1571 a_{v} \approx-0.0076$. These two values of the shift of the freezing volume fraction are within 10\% apart. We therefore believe that "prefreezing" as observed in Refs. 23, 24 is a manifestation of capillary crystallization, and not the equivalent of "layering" or "prewetting" transitions as observed in systems where attractive interactions with the walls are present. ${ }^{3}$ On the other hand, it has recently been observed by computer simulation that if a (single) wall is not smooth but patterned, "surface freezing" occurs at large undersaturation. ${ }^{25}$ In a slit (or any other) geometry this phenomenon may compete with capillary freezing. Which of the two dominates depends on the interfacial tensions of the fluid and the crystal faces with the patterned substrates. It is expected that appropriately patterned surfaces [e.g., surfaces structured in such a way as to fit the (111) crystal face] significantly reduce the crystal-wall interfacial tensions, leading to larger shifts of the freezing density compared to the situation at smooth hard walls.

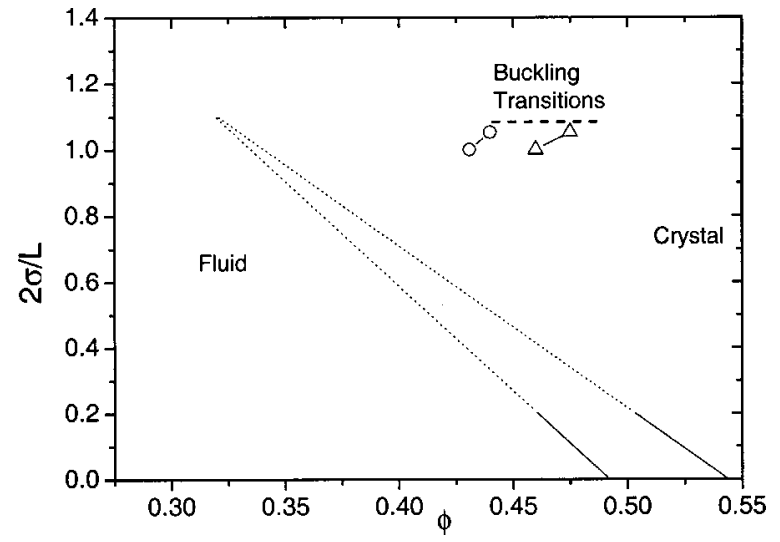

FIG. 3. Coexisting densities of hard spheres in a slit geometry, with $L$ the width of the slit. Lines correspond to the situation where the (111) crystal face is along the walls (see Table I). The left line indicates freezing, and the right one melting. Dotted lines indicate extrapolation beyond the capillary limit, and are only drawn to indicate that if extrapolation was allowed, a critical point appears (i.e., where the lines cross). The points are data from computer simulations by Schmidt and Löwen (Ref. 26) and are connected to guide the eye. Triangles are melting densities, and circles are freezing densities. At very small plate separations, beyond the dashed horizontal line, triple points involving buckling transitions are observed, and fluid-crystal coexistence is moved to much smaller volume fractions.

\section{LARGE AREA TO VOLUME RATIO}

In this section two geometries are discussed: a slit geometry (i.e., two parallel plates), and a box geometry that is shaped in such a way as to fit a close-packed crystal. In the first situation the area to volume ratio becomes large, but the system may in principle remain in the thermodynamic limit. In the second situation, not only geometry plays a role, but due to the exceedingly small number of particles in boxes with large area to volume ratios, fluctuations also become important.

\section{A. Slit geometry}

Schmidt and Löwen ${ }^{26}$ studied hard spheres between plates using both computer simulation and cell theory. They looked at very small plate separations: in between one and two sphere diameters, corresponding to area to volume ratios of order one. From a plate distance of 2.0 down to approximately 1.86 sphere diameters, at increasing sphere density a transition from fluid to a crystal of two hexagonally packed layers is found. This transition is the analog of the bulk freezing transition in this geometry. At even smaller plate separations, a triple point appears involving a buckled crystal phase. At still smaller plate separations, even a rhombic phase appears and the situation becomes quite complicated. For details the reader is referred to Ref. 26.

We took the (two) points from Ref. 26 that correspond to the transition from fluid to the two hexagonally packed layers at the largest plate separation that they studied. These points were determined using Monte Carlo simulation. They are plotted in Fig. 3, together with the melting and freezing lines in the capillary limit, Eqs. (2.11) the values in Table I for the situation that the (111) crystal face contacts the wall. It can be seen from Fig. 3 that if the melting and freezing lines in the capillary limit are extrapolated to very small plate separations, a critical point appears at $2 \sigma / L \approx 1.13$. 
Continuous transitions and critical points associated with them are not expected in symmetry breaking phase transitions $^{27}$ such as the freezing transition. However, at small plate separations, and perpendicular to the plates, a symmetry element is a poorly defined quantity, and true symmetry breaking along that direction does not occur. Clearly, however, Eqs. (2.11) have long ceased to be valid at such small plate separations. Indeed, at plate separations larger than the "extrapolated critical point," the trend from the small plate separations (points in Fig. 3) indicate that the coexistence region shifts to higher volume fractions, and it slightly seems to widen up, contrary to the trend in the capillary limit. The crossover from a decreasing (in density) and narrowing coexistence region to an increasing and widening one, lies in the broad range of roughly $0.2 \leqslant 2 \sigma / L \leqslant 1.0$. It is quite conceivable, however, that more buckling transitions interfere in between plate separations of 2 and three sphere diameters, and also in between three and four and even larger. Therefore the analog of the freezing transition in a slit may intermittently appear and disappear with varying (small) plate separations. In that case the points in Fig. 3 result after more than a single crossover.

Strictly speaking these results do not rule out the existence of a fluid-crystal critical point. If there is a critical point, it should appear before the buckling transitions enter the stage, i.e., at $0.2<2 \sigma / L<1$, see Fig. 3. This is possible if the freezing and melting lines at $2 \sigma / L>0.2$ converge faster than in a linear manner. But this scenario implies that there also is at least one lower critical point in the $2 \sigma / L$-density plane, as the freezing transition appears again at least when $2 \sigma / L=O(1)$. This scenario seems highly implausible, but at this point it cannot be ruled out.

\section{B. Box geometry}

\section{First-order phase transitions of finite systems in the grand ensemble}

In Ref. 28 thermodynamic properties of systems of hard spheres that are confined in small boxes with hard walls are calculated. The experimental equivalent of these systems are finite sized pores. However, in experimental systems at least one of the sides of the boxes is in direct contact with a (macroscopic) bulk system. In other words, the small systems interact with a bath of constant chemical potential. Here, this interaction is modeled by a smooth hard wall. This approximation will become worse if the systems get smaller. This should be kept in mind if the results presented here are compared to experiments on real pores.

The boxes are shaped in such a way that at a certain volume they can accommodate a fcc or hcp crystal of 8 or 27 spheres. Other box geometries were studied in Refs. 29 and 30 .

The volumes of the boxes can be varied isomorphically. The focus in Ref. 28 is on the grand distribution function, i.e., the probability distribution of finding a box ("pore") with $N$ spheres,

$$
P_{N}=\frac{z^{N} q_{N}}{\Xi},
$$

where the reduced activity $z=\left(\sigma^{3} / \Lambda^{3}\right) e^{\beta \mu}$, with $\sigma$ again the diameter of a hard sphere, $\Lambda$ its thermal de Broglie wavelength, $\beta=1 / k T$ and $\mu$ is the chemical potential. $q_{N}$ is the reduced canonical partition function of a system containing $N$ spheres, and $\Xi=\sum_{N=0}^{N_{\max }} z^{N} q_{N}$ is the grand partition function, with $N_{\max }$ the maximum number of spheres that can be crammed into $V$. Two peaks of the grand distribution function at some value of $z$ indicate a first-order phase transition. $^{31}$ In the Appendix it is proved that the reduced canonical partition function of a system of hard spheres is exactly given by the recursive relation,

$$
q_{N}=\frac{v_{0}^{(N-1)}}{N} q_{N-1},
$$

independent of the size of the system or its boundary conditions. In this equation, the reduced available volume $v_{0}^{(N)}$ $=V_{0}^{(N)} / \sigma^{3}$, where $V_{0}^{(N)}$ is the available volume. This quantity is defined as the configurationally averaged volume in a system of $N$ hard spheres that is available for the center of a $(N+1)$ th hard sphere. Equation (3.2) [actually Eq. (A12) in the appendix from which Eq. (3.2) follows] was first derived by Speedy $^{32}$ who used a lattice, and subsequently let the lattice parameter go to zero. In the appendix we present a derivation without using a lattice. In the thermodynamic limit, Eq. (3.2) reduces to the well-known relation between chemical potential and insertion probability that has been obtained from scaled particle theory, ${ }^{33}$ and which is also known as Widom's insertion theorem. ${ }^{34}$ This is also shown in the Appendix.

\section{Shift of coexisting densities in a box geometry}

The hard spheres are contained in boxes with smooth hard walls. Their volumes define $V_{0}^{(0)}$. The geometry of the boxes was chosen in such a way that at certain box volumes, either $N_{\max }=8$ or $N_{\max }=27$ spheres can be close packed in a hep or fcc stacking. This is the situation when $v_{0}^{(0)}$ $=1 / \sqrt{2}\left(N_{\max }=8\right)$ or $v_{0}^{(0)}=4 \sqrt{2}\left(N_{\max }=27\right)$. The volumes of the boxes are expressed relative to the close packed volume, that is, it is defined that $\alpha=v_{0}^{(0)} / 1 / \sqrt{2}$ if $N_{\max }=8$, and $\alpha$ $=v_{0}^{(0)} / 4 \sqrt{2}$ if $N_{\max }=27$.

In Ref. 28, it was shown that if $\alpha>1.6$, the available volume monotonically decreases with the number of spheres. However, as soon as $\alpha<1.5, v_{0}^{(N)}$ goes through a minimum as a function of $N$, the minimum becoming deeper with decreasing $\alpha$. It could be shown that the available volume going through a minimum as a function of the number of spheres corresponds to the small-system analog of the freezing transition of hard spheres. This freezing transition is particularly obvious from two peaks of the grand distribution function, Eq. (3.1), leading to steep changes in the relevant thermodynamic functions that become steeper when the system size is increased from 8 to 27 spheres. The grand distribution functions at the small-system analogs of the freezing transition are shown in Fig. 4. Contrary to the situation in the thermodynamic limit, there are no discontinuities or singularities in the small system thermodynamic functions. The 


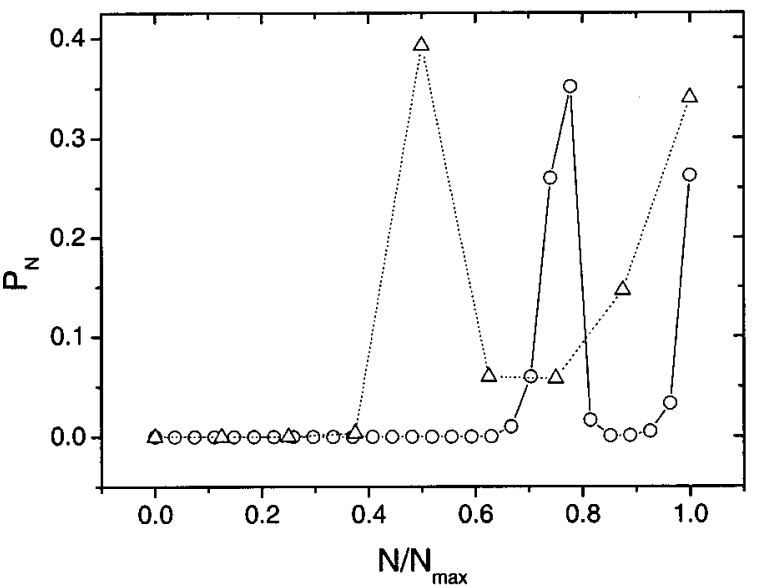

FIG. 4. Grand distribution, Eq. (3.1) at coexistence as a function of $N / N_{\max }$ in systems confined in boxes that may contain at most 8 (triangles plus dotted line) or 27 spheres (circles plus solid line). These two peaks are observed if $\ln (z)=12.45\left(N_{\max }=8\right)$ and $\ln (z)=14.5\left(N_{\max }=27\right)$. In both cases $\alpha=1.3$. Points are connected to guide the eye. Data from Ref. 28 .

reason is that for singularities to exist in the grand ensemble, an infinite number of terms in the grand potential are required. ${ }^{35}$

In the geometry discussed above, the (reduced) area to volume ratio relates to $\alpha$ as $a_{v}=1.624 \alpha^{-1 / 3}$ for $N_{\max }=8$, and $a_{v}=1.112 \alpha^{-1 / 3}$ for $N_{\max }=27$; for details on this and other box geometries, see Ref. 30. In fact, for the purpose of calculating $a_{v}$, the boxes described in Ref. 30 were extended so as to accommodate the whole spheres at close packing and not only their centers with part of the spheres sticking out. The volume fraction in the small systems is defined as

$$
\phi=\frac{N}{N_{\max }} \alpha^{-1} \frac{\pi}{3 \sqrt{2}},
$$

where the numerical factor corresponds to close packing of a fcc or hcp crystal. We took $\alpha=1.3$ for both $N_{\max }=8$ and $N_{\max }=27$. This value of $\alpha$ corresponds to the situation that the systems containing the maximum number of spheres are not close packed, and on the other hand more than $N_{\max }$ spheres cannot be inserted into the systems. In other words, for this value of $\alpha$, the $N_{\max }$ need not be considered as additional constraints imposed on the systems.

In Fig. 5, the points where the small systems freeze and melt are plotted in the area to volume ratio-density plane, together with the melting and freezing lines in the capillary limit, Eqs. (2.11) where the values in Table I for the situation that all three crystal faces (111), (100), and (110) contact the walls in equal amounts. This is a natural choice for a single crystal in the geometry described above. In the thermodynamic limit, only the (111) faces will contact the walls. It is assumed here that on decreasing the system size, defects that arise by "forcing" the crystal to orient along its (111) faces along the walls of the boxes quickly become more expensive than the cost in interfacial free energy by orienting the crystal along two of its nonequilibrium directions. Same as in a slit geometry (Fig. 3), it is clear from Fig. 5 that the extrapolated melting and freezing lines from the capillary limit indicate a critical point (see Sec. III A for a short discussion on

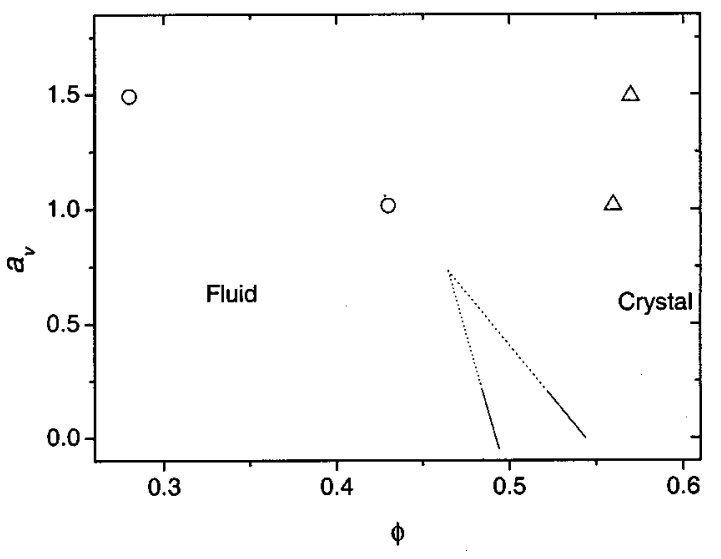

FIG. 5. Coexisting densities of hard spheres in a box geometry. Lines correspond to the situation where the (111), (110), and (100) crystal faces are equally distributed along the walls (see Table I). The left line indicates freezing, and the right one melting. Dotted lines indicate extrapolation beyond the capillary limit, just as in Fig. 3. The points correspond to the peaks of $P_{N}$ in Fig. 4; circles indicate freezing and triangles indicate melting.

critical points in small systems). This point lies at a significantly smaller value of the area to volume ratio than in the slit geometry, i.e., at $a_{v}=0.74$ instead of 1.13 , but still at a value beyond the capillary limit. On the other hand, the fluid-solid coexistence region has become very broad in the small boxes, spanning a volume fraction in between 0.28 and 0.56 for the smallest system (largest $a_{v}$ with $N_{\max }=8$ ). The crossover from converging phase boundaries to diverging ones lies in the very broad interval of roughly $0.2 \leqslant a_{v} \leqslant 1$. Just as in the situation for the slit geometry, in this interval either an upper and a lower critical point exist or no critical point at all. Contrary to the slit geometry, fluctuations become important due to the smallness of the systems. This issue is addressed in the next section.

\section{Role of fluctuations}

At a first order phase transition in the thermodynamic limit, two densities coexist and $P_{N}$ has two delta peaks, both centered at the average number of particles in the coexisting phases (at fixed system volume), further referred to as $\left\langle N_{1}\right\rangle$ and $\left\langle N_{2}\right\rangle$. In this limit, the variance of the number of particles can immediately be calculated, with the result

$$
\sigma_{N}^{2}=\sum_{N=0}^{\infty} N^{2} P_{N}-\left(\sum_{N=0}^{\infty} N P_{N}\right)^{2}=\frac{1}{4}\left(\left\langle N_{2}\right\rangle-\left\langle N_{1}\right\rangle\right)^{2}
$$

so that the relative magnitude of the fluctuations become

$$
\frac{\sigma_{N}}{\langle N\rangle}=\frac{\left|\left(\left\langle N_{2}\right\rangle-\left\langle N_{1}\right\rangle\right)\right|}{\left(\left\langle N_{2}\right\rangle+\left\langle N_{1}\right\rangle\right)}=\frac{\left|\phi_{2}-\phi_{1}\right|}{\phi_{2}+\phi_{1}}=|\Delta \phi| /\left(\phi_{1}+\phi_{2}\right),
$$

since obviously, the average number of particles in the whole system is given by $\langle N\rangle=\left(\left\langle N_{1}\right\rangle+\left\langle N_{2}\right\rangle\right) / 2$. Equation (3.4) is exact in the thermodynamic limit where it is a constant as the two phases contain constant volume fractions $\phi_{i}$.

Now if the systems become smaller, fluctuations will come into play and the two individual delta peaks will get 
finite widths, as in Fig. 4. This width of the individual peaks is quantified by $\sigma_{N_{\alpha}}$, being the variance in the number of particles in phase $\alpha, N_{\alpha}$. In general, ${ }^{31}$

$$
\sigma_{N_{\alpha}} /\left\langle N_{\alpha}\right\rangle=A(\phi) / \sqrt{N_{\alpha}},
$$

where $A(\phi)=(\partial \widetilde{p} / \partial \phi)^{-1 / 2}$ only depends upon the volume fraction of hard spheres in the system. It should be noted that in this equation, $\left\langle N_{\alpha}\right\rangle$ and $N_{\alpha}$ refer to the number of particles in one of the coexisting phases in the system, whereas in Eq. (3.4), $\langle N\rangle$ is the average in the whole system. Equation (3.5) implies that if the system size is reduced in such a way that the volume fraction remains constant, the two individual peaks broaden as $1 / \sqrt{N_{\alpha}}$. At some point these peaks will overlap, and the two states can no longer be distinguished. In other words, the phase transition is killed by fluctuations. This is where $\left(\sigma_{N} /\langle N\rangle\right) \approx\left(\sigma_{N_{\alpha}} /\left\langle N_{\alpha}\right\rangle\right)$.

Does this happen in the systems studied here? It is obvious from Fig. 4 that even in a very small system that contains at most 8 spheres, the width of the coexistence region far exceeds the broadness of the individual peaks, i.e., $\left(\sigma_{N} /\langle N\rangle\right) \gg\left(\sigma_{N_{\alpha}} /\left\langle N_{\alpha}\right\rangle\right)$. But the width of the coexistence region steeply increases on decreasing the system size from $N_{\max }=27$ to $N_{\max }=8$. It might thus be possible that fluctuations kill the freezing transition somewhere in the broad range of system sizes in between $N_{\max }=27$, and sizes within the capillary limit. We therefore address the question as to how the width of the coexistence region competes with the widths of the individual peaks, in other words, how $\left(\sigma_{N} /\langle N\rangle\right)$ relates to $\left(\sigma_{N_{\alpha}} /\left\langle N_{\alpha}\right\rangle\right)$ as a function of system size.

In the capillary limit, we just use Eq. (3.4) together with the information on the average crystal orientation as listed in Table I. This is equivalent to transforming the lines in Fig. 5 into a single line. In calculating the individual peakwidths, only the fluid branch is considered, i.e., $\left(\sigma_{N_{f}} /\left\langle N_{f}\right\rangle\right)$ is calculated, as the bulk moduli of fluid and solid are of the same order of magnitude. First of all we eliminate $N_{f}$ in Eq. (3.5). For this purpose use is made of Eq. (3.3). Subsequently we relate $N_{\max }$ to the area to volume ratio of the particular geometry studied here. This relation, for fixed $\alpha=1.3$ reads $^{30}$ $N_{\max } \approx 27.44 a_{v}^{-3}$, so that $N_{f} \approx 48.17 a_{v}^{-3} \phi$. The quantity $A(\phi)=(\partial \widetilde{p} / \partial \phi)^{-1 / 2}$ in Eq. (3.5) is extracted from the fluid branch of the Hall equation of state, Eq. (2.13), expanded up to first order around $\phi_{f}^{*}$ with the result that $A(\phi) \approx 0.137$ $-0.67\left(\phi-\phi_{f}^{*}\right)$. Combining all this leads to

$$
\begin{aligned}
\sigma_{N_{f}} /\left\langle N_{f}\right\rangle=A(\phi) / \sqrt{N_{f}} \approx & \left(0.137-0.67\left(\phi-\phi_{f}^{*}\right)\right) \\
& \times\left(\frac{48.17 \phi}{a_{v}^{3}}\right)^{-1 / 2},
\end{aligned}
$$

where it is again emphasized that the numerical factor in the last term in this expression depends upon geometry. Substituting Eq. (2.11) for $\phi=\phi_{f}^{*}+\Delta \phi$, and making use of the quantities in Table I finally leads to the relation between the width of the individual fluid peak in the grand distribution, and the area to volume ratio of the system. Equations (3.4) and (3.6) are plotted in Fig. 6, again for the situation where all crystal faces are equally distributed along the walls. The

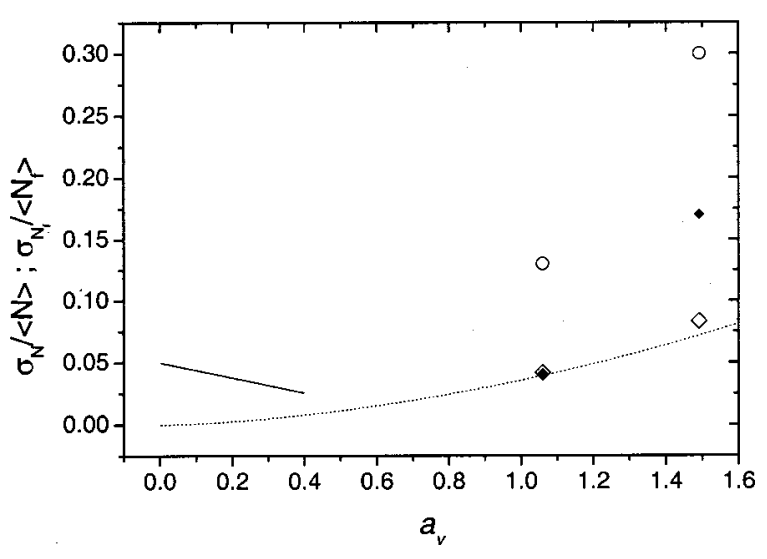

FIG. 6. Fluctuations in the coexistence region of the freezing transition, where the hard spheres are confined in boxes (see text). The solid line are the fluctuations within the capillary limit, i.e., Eq. (3.4) together with Eqs. (2.11) and Table I for the average crystal orientation (see text). Circles correspond to the small systems. The dotted line are the fluctuations of the individual fluid peaks, Eq. (3.6) together with Eq. (2.11) and Table I. Open diamonds are extracted from the widths of the fluid peaks in Fig. 4. Solid diamonds are calculated using Eq. (3.6), but using the volume fractions of the fluid peaks that correspond to the ones in Fig. 5.

point at which they cross indicate where the phase transition is "killed" by fluctuations. First of all it is obvious from Fig. 6 that fluctuations only become significant beyond the capillary limit, i.e., when $a_{v}>0.2$. If extrapolation beyond this limit was allowed, the lines would cross at $a_{v} \approx 0.6$; it should be noted that the solid line has already been drawn beyond the capillary limit. This value is significantly smaller than the (also extrapolated) value of $a_{v} \approx 0.74$, where the two phase boundaries cross in Fig. 5. We also plotted the values of $\sigma_{N} /\langle N\rangle$ and $\sigma_{N_{f}} /\left\langle N_{f}\right\rangle$ obtained from the systems in the small boxes. The first quantities were calculated using $\sigma_{N}^{2}$ $=\Sigma_{N=0}^{N_{\max }} N^{2} P_{N}-\left(\Sigma_{N=0}^{N_{\max }} N P_{N}\right)^{2}$. It is worth mentioning that these values are in very good agreement with Eq. (3.4) (within $10 \%$ ), see again Fig. 6 . They clearly are much larger than the ones that are extrapolated from the capillary limit, which already is obvious from Fig. 5. The widths of the individual peaks of $P_{N}$ in Fig. 4 were calculated by cutting them off at $N=6\left(N_{\max }=8\right)$ and $N=24\left(N_{\max }=27\right)$. As can be seen in Fig. 6, they are in good agreement with the ones calculated by Eq. (3.6). We also calculated $\sigma_{N_{f}} /\left\langle N_{f}\right\rangle$ substituting the observed freezing volume fractions $\phi=0.43$ and $\phi=0.28$ into Eq. (3.6) for the systems with $N_{\max }=27$ and $N_{\max }=8$, respectively. These results are also shown in Fig. 6 (as filled symbols). Clearly in the first case quantitative agreement is observed, but in the second case Eq. (3.6) predicts too large a value for the fluctuations.

We conclude from this analysis that fluctuations in these small systems play no significant role in the capillary limit. They become important when $a_{v}$ is larger than roughly 0.2. When $a_{v}$ is of order 1 , phase boundaries diverge while in the capillary limit they converge. On the other hand, number fluctuations within the coexisting phases are in very good agreement with predictions using the macroscopic Hall equation of state together with the capillary limit Eqs. (2.11). If the phase boundaries had not widened at large values of $a_{v}$, the freezing transition would have been killed by fluctuations 
when $a_{v} \approx 0.6$, see Fig. 6 . This number corresponds to a system that can contain approximately 127 spheres. Now depending upon how $\sigma_{N} /\langle N\rangle$ crosses over from decreasing linearly with $a_{v}$ (capillary limit) to steeply increasing when $a_{v}$ becomes of order 1, there are two scenarios. The first is that $\sigma_{N} /\langle N\rangle$ crosses $\sigma_{N_{f}} /\left\langle N_{f}\right\rangle$, so that the freezing transition is killed by fluctuations. This might in principle happen in the interval of roughly $0.6 \leqslant a_{v} \leqslant 0.9$, corresponding to systems that may contain in between approximately 40 and 130 spheres. The second scenario is that this crossover sets in before roughly $a_{v} \approx 0.6$. In that case the (small system analog of the) freezing transition will take place in the whole range of macroscopically large systems down to the very small ones as studied here.

\section{CONCLUDING REMARKS}

In this work we have addressed the question as to what confinement does to the freezing transition of hard spheres. We have provided an answer to this question in the capillary limit, as well as in the limit that systems are very small. In the first case, we showed that hard spheres in confining geometries will freeze at densities significantly below the bulk freezing density. This implies that if a porous system is in contact with bulk, the part of the system in the pores will freeze, while the bulk remains fluid. In the second limit the answer necessarily cannot be general as geometry becomes a determining factor. The question remains what happens in between these limits, as in this range critical points may occur, and fluctuations may kill the phase transition. This is not a simple question to answer using classical statistical thermodynamics. The existence of the expansion of the free energy in powers of the area to volume ratio [i.e., Eqs. (2.6)] already is questionable if terms of quadratic order need to be taken into account. It will definitely not exist for even higher order. The physical reason for this is that the bulk reference free energy, i.e., the zeroth-order term in the area to volume ratio, $a_{v}$, will become itself a function of system size and geometry. Therefore, one should either look for another reference state, or rely on direct computer simulation or density functional techniques in order to study the intermediate range of $a_{v}$. The latter technique was applied to study wetting behavior of molecular fluids in pores, see Ref. 5 for a review.

In small systems, but probably also in the capillary limit, it may become exceedingly expensive for the system to have two coexisting phases within the same pore. For this reason it is expected that at coexistence, in a collection of pores part of them will contain (single phase) fluid, while the other fraction contains (single phase) crystal. Within a single pore, the system may oscillate between the fluid and crystalline state.

In order to compare to experiments, the question of thermodynamic equilibrium should be addressed. In systems of bulk hard sphere colloids, quenched nonequilibrium states may only very slowly relax, see, for example Refs. 36,37 . It is expected that these relaxations will become even slower in confined systems. Therefore metastable states may confound the scenarios as sketched in this work.
When pores become small, their detailed geometry is expected to be important. A pore geometry that may be particularly interesting is one that is just slightly deformed with respect to the ones that were studied under the "box geometry," Sec. III B. In a particular case, freezing was observed to occur in two steps. ${ }^{30}$ It would be intriguing to see this scenario verified in real systems.

\section{ACKNOWLEDGMENTS}

Correspondence with Hartmut Löwen, and discussions with Jan Groenewold, Marjolein Dijkstra, Howard Reiss, Daan Frenkel, Bob Evans, and Alfons van Blaaderen are gratefully acknowledged. Jan Groenewold and Roel Dullens are thanked for comments on the manuscript.

\section{APPENDIX: RECURSIVE RELATION BETWEEN PARTITION FUNCTION AND AVAILABLE VOLUME FOR HARD SPHERES}

The probability that a sequence of $N$ randomly (and uniformly) generated coordinates of hard sphere centers in a volume $V$ (defined here as the volume available for a single sphere center), have a configuration such that none of the hard spheres overlap, and where one sphere center is in $d r_{1}$ at $r_{1}$, a second is in $d r_{2}$ at $r_{2}, \ldots$, and an $N$ th is in $d r_{N}$ at $r_{N}$, is the insertion probability of such a configuration. It reads

$$
P_{\mathrm{ins}}\left(r_{1}, \ldots, r_{N}\right)=\frac{\left(\Pi_{i<j} \delta\left(\widetilde{r}_{i j}\right)\right) d r_{1} \cdots d r_{N}}{\int_{V} \cdots \int d r_{1} \cdots d r_{N}},
$$

with

$$
\widetilde{r}_{i j}=\frac{\left|r_{i}-r_{j}\right|}{\delta},
$$

where $\delta$ denotes the diameter of a sphere. Furthermore,

$$
\begin{aligned}
& \delta\left(\widetilde{r}_{i j}\right)=0\left(\widetilde{r}_{i j}<1\right), \\
& \delta\left(\widetilde{r}_{i j}\right)=1\left(\widetilde{r}_{i j} \geqslant 1\right) .
\end{aligned}
$$

The insertion (or acceptance) probability averaged over all sets of coordinates within $V$ is

$$
\left\langle P_{\text {ins }}(N)\right\rangle=\frac{\int_{V} \cdots \int\left(\Pi_{i<j} \delta\left(\widetilde{r}_{i j}\right)\right) d r_{1} \cdots d r_{N}}{\int_{V^{*}} \cdot \int d r_{1} \cdots d r_{N}}=\frac{Z_{N}}{V^{N}},
$$

with $Z_{N}$ the configuration integral of $N$ hard spheres in volume $V$. This is easily proved as follows.

A general expression for the configuration integral of $N$ particles in a volume $V$ is

$$
Z_{N}=\int_{V} \cdots \int e^{-\beta U\left(r_{1} \cdots r_{N}\right)} d r_{1} \cdots d r_{N},
$$

where $U\left(r_{1}, \ldots, r_{N}\right)$ denotes the potential energy of the system, which, for hard spheres, is rigorously pairwise additive, i.e.,

$$
U\left(r_{1}, \ldots, r_{N}\right)=\sum_{i<j} u\left(\widetilde{r}_{i j}\right),
$$

with the pair potential, 


$$
\begin{aligned}
& u\left(\tilde{r}_{i j}\right)=\infty\left(\tilde{r}_{i j}<1\right), \\
& u\left(\widetilde{r}_{i j}\right)=0\left(\widetilde{r}_{i j} \geqslant 1\right),
\end{aligned}
$$

so that,

$$
e^{-\beta U\left(r_{1} \cdots r_{N}\right)}=e^{-\mu \Sigma_{i<j} u\left(\tilde{r}_{i j}\right)}=\prod_{i<j} \delta\left(\tilde{r}_{i j}\right),
$$

which completes the proof.

In principle, by measuring the average insertion probability Eq. (A4), the configuration integral follows. However, even for moderately dense, and still rather small systems, $\left\langle P_{\text {ins }}(N)\right\rangle$ rapidly goes to zero. Fortunately $\left\langle P_{\text {ins }}(N)\right\rangle$ can be factored as

$$
\begin{aligned}
\left\langle P_{\text {ins }}(N)\right\rangle= & \left\langle P_{\text {ins }}(1) P_{\text {ins }}(2 \mid 1) P_{\text {ins }}(3 \mid 1,2) \cdots\right. \\
& \left.\times P_{\text {ins }}(N \mid 1,2, \ldots,(N-1))\right\rangle,
\end{aligned}
$$

where $P_{\text {ins }}(j \mid 1,2, \ldots,(j-1))$ is shorthand for the probability of accepting particle $j$ at $\mathbf{r}_{j}$ provided that $(j-1)$ sphere centers are at $\left\{\mathbf{r}_{1} \cdots \mathbf{r}_{(j-1)}\right\}$. The next step is the crucial one. For hard spheres, the average of all possible insertion sequences equals the sequence of averages,

$$
\begin{aligned}
& \left\langle P_{\text {ins }}(1) P_{\text {ins }}(2 \mid 1) \cdots P_{\text {ins }}(N \mid 1,2, \ldots,(N-1))\right\rangle \\
& \quad=\left\langle P_{\text {ins }}(1)\right\rangle\left\langle P_{\text {ins }}(2 \mid 1)\right\rangle \cdots\left\langle P_{\text {ins }}(N \mid 1,2, \ldots,(N-1))\right\rangle .
\end{aligned}
$$

On the right-hand side (rhs) of Eq. (A10), the terms are averages over all possible configurations, in other words, the insertion probability $\left\langle P_{\text {ins }}(N \mid 1,2, \ldots,(N-1))\right\rangle$ is the insertion probability of the $N$ th sphere into a system containing $(N$ -1 ) spheres, averaged over all possible configurations of the $(N-1)$ spheres. The terms on the rhs of Eq. (A10) relate to the available volume via

$$
V_{0}^{(N)}=\left\langle P_{\text {ins }}(N+1 \mid 1,2, \ldots, N)\right\rangle V,
$$

that is, the insertion probability of an $(N+1)$ th sphere center into a system of $N$ hard spheres is just the ratio of the available volume of the system of $N$ hard spheres and the available volume for a single sphere without any others present.

Combining Eqs. (A9)-(A11) with Eq. (A4) gives

$$
Z_{N}=\prod_{J=0}^{N-1} V_{0}^{(J)}
$$

with $V_{0}^{(0)}=V$ in our definition. Equation (A12) has for the first time been obtained by Speedy, ${ }^{32}$ who used a lattice and subsequently let the lattice parameter go to zero.

Equation (A12) can be written as a recursive relation,

$$
Z_{N}=V_{0}^{(N-1)} Z_{N-1} \text {, }
$$

and via the definition of the canonical partition function,

$$
Q_{N}=\frac{Z_{N}}{\Lambda^{3 N} N !},
$$

with $\Lambda$ the thermal De Broglie wavelength, it is easily seen that the partition function may also be written as a recursive relation, i.e.,

$$
Q_{N}=\frac{V_{0}^{(N-1)}}{\Lambda^{3} N} Q_{N-1} .
$$

With $q_{N}=\left(\Lambda^{3} / \sigma^{3}\right) Q_{N}$, Eq. (A15) is seen to resemble Eq. (3.2). It should be noted that no assumptions have been made regarding the size or shape of the system of hard spheres. All the above equations therefore apply to hard sphere systems of arbitrary size, and with arbitrary boundary conditions [implicit in the available volume of "empty" space, $V$, which resembles here $\left.V_{0}^{(0)}\right]$.

We will now show that, starting from Eq. (A15) we recover a well-known relation by scaled particle theory, ${ }^{33}$ and by Widom ${ }^{34}$ between insertion probability and chemical potential. It is shown that the latter relation only holds for a system in the thermodynamic limit.

Equation (A15) may be written as

$$
\frac{V_{0}^{(N)}}{\Lambda^{3}(N+1)}=\frac{Q_{N+1}}{Q_{N}},
$$

or,

$$
\ln \left(\frac{V_{0}^{(N)}}{\Lambda^{3}(N+1)}\right)=\ln Q_{N+1}-\ln Q_{N}=\beta\left(F_{N}-F_{N+1}\right),
$$

with $F_{N}$ the Helmholz free energy of a system containing $N$ hard spheres. The chemical potential is defined as

$$
\mu=\left(\frac{\partial F}{\partial N}\right)_{T V} .
$$

Comparing Eqs. (A17) and (A16), and noting that

$$
\begin{aligned}
& \lim \left(F_{N+1}-F_{N}\right) / 1\left(\frac{\partial F}{\partial N}\right)_{T V} \\
& N \rightarrow \infty,
\end{aligned}
$$

we immediately identify

$$
\beta \mu=-\ln \left(\frac{V_{0}^{(N)}}{\Lambda^{3}(N+1)}\right) \quad[N \rightarrow \infty] .
$$

With negligible error, in the limit $N \rightarrow \infty$, Eq. (A19) can be written as

$$
\beta \mu=-\ln \left(\frac{V_{0}^{(N)}}{\Lambda^{3} N}\right) .
$$

This relation follows from scaled particle theory. ${ }^{33}$ Since the insertion probability of an $(N+1)$ th hard particle in a system of $N$ hard particles is $P_{\text {ins }}=V_{0}^{(N)} / V$, Eq. (A20) can be seen to resemble the well known general expression of the chemical potential obtained by Widom. ${ }^{34}$ Clearly, it is an asymptotic relation that does not apply to small systems.

${ }^{1}$ K. Huang, Statistical Mechanics, 2nd ed. (Wiley, New York, 1987)

${ }^{2}$ P. G. Debenedetti, Metastable Liquids (Princeton University Press, Princeton, 1996).

${ }^{3}$ S. Dietrich, in Phase Transitions and Critical Phenomena (Academic, London, 1988), pp. 1-128.

${ }^{4}$ W. J. Huisman, J. F. Peters, M. J. Zwanenburg, S. A. deVries, T. E. Derry, D. Abernathy, and J. F. van der Veen, Nature (London) 390, 379 (1997).

${ }^{5}$ R. Evans, J. Phys.: Condens. Matter 2, 8989 (1990).

${ }^{6}$ M. M. Kohonen, N. Maeda, and H. K. Christenson, Phys. Rev. Lett. 82, 4667 (1999).

${ }^{7}$ E. Molz, A. P. Y. Wong, M. H. W. Chan, and J. R. Beamish, Phys. Rev. B 48, 5741 (1993).

${ }^{8}$ H. K. Christenson, Phys. Rev. Lett. 74, 4675 (1995). 
${ }^{9}$ L. D. Gelb, K. E. Gubbins, R. Radhakrishnan, and M. SliwinskaBartkowiak, Rep. Prog. Phys. 62, 1573 (1999).

${ }^{10}$ H. K. Christenson, J. Phys.: Condens. Matter 13, R95 (2001).

${ }^{11}$ B. J. Alder and T. E. Wainwright, J. Chem. Phys. 27, 1208 (1957).

${ }^{12}$ W. W. Wood and J. D. Jacobsen, J. Chem. Phys. 27, 1207 (1957).

${ }^{13}$ W. G. Hoover and F. H. Ree, J. Chem. Phys. 49, 3609 (1968).

${ }^{14}$ P. N. Pusey and W. vanMegen, Nature (London) 320, 340 (1986).

${ }^{15}$ W. C. K. Poon, P. N. Pusey, and H. N. W. Lekkerkerker, Phys. World 9, 27 (1996).

${ }^{16}$ R. Evans and U. M. B. Marconi, J. Chem. Phys. 84, 2376 (1986).

${ }^{17}$ R. Defay, I. Prigogine, and A. Bellemans, Surface Tension and Adsorption (Longmans Green, London, 1966).

${ }^{18}$ K. R. Hall, J. Chem. Phys. 57, 2252 (1972).

${ }^{19}$ D. Frenkel and B. Smit, Understanding Molecular Simulation (Academic, London, 1996).

${ }^{20}$ H. Reiss, H. L. Frich, E. Helfand, and J. L. Lebowitz, J. Chem. Phys. 32, 119 (1960).

${ }^{21}$ M. Heni and H. Löwen, Phys. Rev. E 60, 7057 (1999).

${ }^{22}$ J. Klein and E. Kumacheva, Science 269, 816 (1995).
${ }^{23}$ D. J. Courtemanche and F. van Swol, Phys. Rev. Lett. 69, 2078 (1992).

${ }^{24}$ D. J. Courtemanche, T. A. Pasmore, and F. van Swol, Mol. Phys. 80, 861 (1993).

${ }^{25}$ M. Heni and H. Löwen, Phys. Rev. Lett. 85, 3668 (2000).

${ }^{26}$ M. Schmidt and H. Löwen, Phys. Rev. E 55, 7228 (1997).

${ }^{27}$ L. D. Landau and E. M. Lifshitz, Statistical Physics, 3rd ed. (Pergamon, New York, 1980).

${ }^{28}$ W. K. Kegel, H. Reiss, and H. N. W. Lekkerkerker, Phys. Rev. Lett. 83, 5298 (1999).

${ }^{29}$ Z. T. Németh and H. Löwen, Phys. Rev. E 59, 6824 (1999).

${ }^{30}$ W. K. Kegel, Phys. Rev. E 63, 037104 (2001).

${ }^{31}$ T. L. Hill, Statistical Mechanics (Dover, New York, 1987).

${ }^{32}$ R. J. Speedy, J. Chem. Soc., Faraday Trans. 2 73, 714 (1977).

${ }^{33}$ H. Reiss and A. D. Hammerich, J. Phys. Chem. 90, 6252 (1986).

${ }^{34}$ B. Widom, J. Chem. Phys. 39, 2802 (1963).

${ }^{35}$ C. N. Yang and T. D. Lee, Phys. Rev. 87, 404 (1952).

${ }^{36}$ S. Pronk and D. Frenkel, J. Chem. Phys. 110, 4589 (1999).

${ }^{37}$ W. K. Kegel and J. K. G. Dhont, J. Chem. Phys. 112, 3431 (2000). 